\title{
Silicon nanocluster-sensitized emission from erbium: The role of stress in the formation of silicon nanoclusters
}

\author{
I. Ahmad, ${ }^{1}$ M. P. Temple, ${ }^{2}$ A. Kallis, ${ }^{1}$ M. Wojdak, ${ }^{1}$ C. J. Oton, ${ }^{2}$ D. Barbier, ${ }^{3}$ H. Saleh, ${ }^{1}$ \\ A. J. Kenyon, ${ }^{1, a)}$ and W. H. Loh ${ }^{2}$ \\ ${ }^{1}$ Department of Electronic and Electrical Engineering, University College London, WC1E 7JE London, \\ United Kingdom \\ ${ }^{2}$ Optoelectronics Research Centre, University of Southampton, SO17 1BJ Southampton, United Kingdom \\ ${ }^{3}$ Teem Photonics, 61, Chemin du Vieux Chêne 38246 Meylan, France
}

(Received 3 September 2008; accepted 6 November 2008; published online 18 December 2008)

\begin{abstract}
Erbium-doped silicon-rich silicon oxide films deposited by plasma enhanced chemical vapor deposition suffer from compressive stress as deposited, which converts to a large tensile stress on annealing due to the release of hydrogen. Although the cracking that results from this stress can be avoided by patterning the films into ridges, significant stress remains along the ridge axis. Measurements of erbium photoluminescence sensitized by silicon nanoclusters in stressed and relaxed films suggest an important role for internal film stresses in promoting the phase separation of excess silicon into nanoclusters, which has previously been thought of as a thermally driven process. (C) 2008 American Institute of Physics. [DOI: 10.1063/1.3050324]
\end{abstract}

\section{INTRODUCTION}

Silicon-rich silicon oxide [or silica containing silicon nanoclusters (Si-ncs) $],{ }^{1}$ has been the subject of considerable attention for optoelectronic applications. Significantly, in combination with erbium as a dopant, the Si-ncs have been found to serve as effective sensitizers, producing enhanced Er photoluminescence (PL) and electroluminescence ${ }^{2-4}$ at the important telecommunications wavelengths around $1.55 \mu \mathrm{m}$. As a result, this material has been suggested as a promising candidate for silicon-based lasers or optical amplifiers. 5

The sensitization process in this material remains relatively poorly understood, although it is generally agreed that a high degree of control over the size distribution, density and crystallinity of Si-ncs is required to fully optimize the material parameters. Conventionally, cluster size distribution and density have been controlled by varying either the excess silicon concentration and/or annealing conditions. ${ }^{6-8}$ It is assumed that the growth and phase separation of Si-ncs is driven by annealing. ${ }^{9}$ However, we present results in this paper that point to a significant role for film stress in controlling the formation of nanoclusters.

Control of film stress in optical materials is critical for a variety of reasons, including the avoidance of cracking and delamination of films from substrates. It is well known that thick $(>500 \mathrm{~nm})$ plasma enhanced chemical vapor deposition (PECVD) $\mathrm{SiO}_{x}$ films can suffer from severe microcracking, which occurs during high temperature annealing. ${ }^{10-12}$ Under thermal treatment, the film shrinks due to removal of the hydrogen incorporated into the film during the deposition process. ${ }^{10,13-15}$

Conventional silane-based PECVD of silicon dioxide exploits the plasma dissociation of silane $\left(\mathrm{SiH}_{4}\right)$ and nitrous

\footnotetext{
${ }^{a)}$ Author to whom correspondence should be addressed. Electronic mail: t.kenyon@ee.ucl.ac.uk.
}

oxide $\left(\mathrm{N}_{2} \mathrm{O}\right)$ to form silicon and oxygen reactive species (ions and radicals) that react on the substrate surface to produce films of $\mathrm{SiO}_{2}$. By controlling the flow rates of the precursor gases, the film stoichiometry is continuously variable over a wide range of compositions. Thus, for example, silicon-rich material may be produced by increasing the silane flow to generate an excess of silicon in the deposited material. Under ideal conditions, silane will dissociate fully in the plasma according to

$$
e^{-}+\mathrm{SiH}_{4} \rightarrow \mathrm{Si}+2 \mathrm{H}_{2}+e^{-} .
$$

However, the plasma chemistry is complex, and there is a large number of dissociative pathways available to silane, of which the most likely is ${ }^{16}$

$$
e^{-}+\mathrm{SiH}_{4} \rightarrow \mathrm{SiH}_{3}+\mathrm{H}+e^{-} \text {. }
$$

Hence, silica films grown from plasma dissociation of silane are generally rich in hydrogen. Moreover, increasing the silicon content of the film also increases the hydrogen concentration. The presence of hydrogen in the films leads to compressive stress as the film is prevented from expanding by the substrate. Annealing at temperatures in excess of $600^{\circ} \mathrm{C}$ is sufficient to remove the incorporated hydrogen, ${ }^{10,17}$ after which the film experiences tensile stress as the silica network relaxes. Experimental measurements of the fracture strength of PECVD silica films ${ }^{18}$ indicate stresses in excess of $360 \mathrm{MPa}$ are sufficient to cause fracture of thick $(1 \mu \mathrm{m})$ films. The problem becomes increasingly severe as the $\mathrm{SiO}_{x}$ films are made thicker (higher stress buildup) or more Si-rich (the higher silane fluence during growth results in more hydrogen and more stress).

The potential for internal stresses to cause fracture of the film also depends on the film adhesion to the substrate. ${ }^{19}$ Strong adhesion may be achieved with, for example, a plasma treatment of the substrate before the film deposition step, but this can result in the cracks propagating into the substrate. ${ }^{12}$ In atomic force microscopy (AFM) studies of our 
films, it was seen that some cracks were more than $2.2 \mu \mathrm{m}$ deep, which was the limit of the AFM capability. This is greater than the film thickness. Without the substrate plasma treatment, adhesion is reduced and the film will crack and separate from substrate, leaving the substrate intact.

Film stress can be minimized by growing thin films $(\sim 200 \mathrm{~nm}$ ), but this will significantly limit the range of potential applications. For instance, for optical waveguides for use around $\lambda=1.5 \mu \mathrm{m}$, a film thickness of about $1 \mu \mathrm{m}$ would be typically required. A better approach is to etch the deposited films into the desired microstructure, e.g., a series of ridges for optical waveguides, prior to annealing. This allows room for the etched film sections to relax during annealing, preventing crack formation and film delamination. Griffith fracture theory states that the critical fracture stress of a thin ridge of material increases as the width of the ridge decreases. However, it should be borne in mind that significant uniaxial stress still exists along the axis of the etched ridges, despite stress relaxation in the perpendicular direction. As we find below, the stresses within these PECVDgrown films have consequences other than simple film fracture-stress affects the nucleation of Si-ncs, and the effect is manifested in the PL behavior of the material. In Sec. II we first characterize the mechanical consequences of film stress-fracture, delamination-and show how these can be minimized; in Sec. III we present results that show that even if fracture is avoided, the residual stress can be sufficient to drastically modify the material structure, and the ensuing PL behavior, of these films.

\section{STRESS-INDUCED CRACKING OF FILMS}

A series of test samples were deposited with thicknesses up to $2 \mu \mathrm{m}$ and varying silicon excesses-from nearly stoichiometric to moderately silicon rich (approximately 10\% excess silicon). Samples were grown by PECVD in an Oxford Instruments DP80 capacitively coupled deposition system. $\mathrm{SiO}_{x}$ : Er layer thicknesses between 1 and $2 \mu \mathrm{m}$ were deposited on $\mathrm{Si}$ (100) substrates both with and without a $5 \mu \mathrm{m}$ oxide. Silane $\left(\mathrm{SiH}_{4}\right)$ and nitrous oxide $\left(\mathrm{N}_{2} \mathrm{O}\right)$ were used as precursor gases - the former being diluted to $5 \%$ in nitrogen - and erbium(thd $)_{3}$ vapor with argon as a carrier gas was used to achieve erbium doping during the deposition. Postdeposition anneals in a tube furnace were carried out either at 900 or $1100{ }^{\circ} \mathrm{C}$ in an argon ambient to precipitate and grow $\mathrm{Si}$ nanoclusters. Images of the samples were taken by optical microscope and AFM, and Fourier transform infrared (FTIR) spectra were measured to determine the hydrogen content of the films. We estimate the concentrations of erbium and Si-ncs in the films to be approximately $10^{19}$ and $10^{17} \mathrm{~cm}^{-3}$, respectively.

Selected samples were plasma etched before annealing using standard photolithography and $\mathrm{SF}_{6}$ (sulfur hexafluoride) to produce a series of ridge structures with ridge widths varying between 0.6 and $20 \mu \mathrm{m}$ and ridge heights of approximately $0.5-1 \mu \mathrm{m}$. The samples were annealed after etching using the same protocol as the unetched samples.

Microscope images of annealed $1 \mu \mathrm{m}$ thick $\mathrm{SiO}_{x}$ : $\mathrm{Er}$ films are shown in Fig. 1. In these cases, the changes in

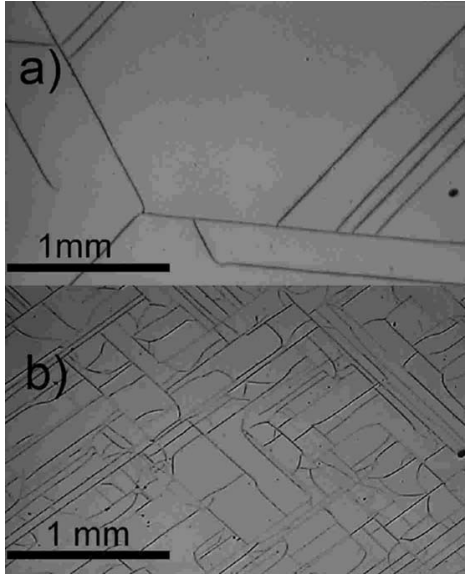

FIG. 1. Optical microscope images of cracked Er: SRSO films: (a) $500 \mathrm{~nm}$ thick film; (b) $1 \mu \mathrm{m}$ thick film. Both films have been annealed at $900{ }^{\circ} \mathrm{C}$ in argon for $90 \mathrm{~min}$.

stress within the film during annealing are already sufficient to cause extensive cracking. Cracking occurs rapidly on annealing; even films annealed for times as short as 1 min were cracked. The cracking becomes more extensive with thicker layers, as can be seen in Figs. 1(a) and 1(b) showing $500 \mathrm{~nm}$ and $1 \mu \mathrm{m}$ thick layers respectively. FTIR measurements further confirmed that temperatures of $900{ }^{\circ} \mathrm{C}$ were sufficient to remove hydrogen from the films.

Figure 2 shows surface interferometry measurements on a $1 \mu \mathrm{m}$ thick film deposited on a $500 \mu \mathrm{m}$ thick silicon substrate. The radius of curvature of the sample was found to be $12 \mathrm{~m}$ before annealing (convex profile) and $-12.4 \mathrm{~m}$ after annealing (i.e., changed to a concave profile). Using the Stoney equation ${ }^{20}$ the value of the stress in the film can be calculated,

$$
\sigma_{f}=\frac{E_{s} h_{s}^{2}}{6 h_{f}\left(1-\nu_{s}\right) K},
$$

where $\sigma_{f}$ is the film stress, $E_{s}$ is Young's modulus of silicon, $h_{s}$ is the substrate thickness, $h_{f}$ is the film thickness, $v_{s}$ is Poisson's ratio for silicon, and $K$ is the radius of curvature.

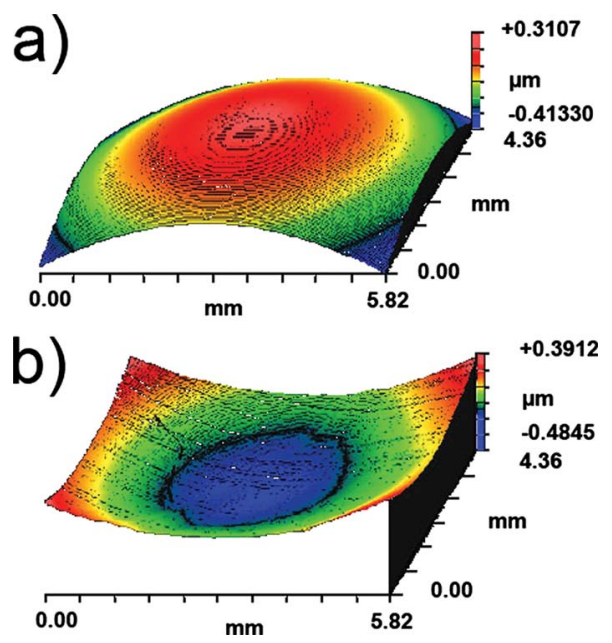

FIG. 2. (Color online) Surface interferometry measurements of a sample (a) before and (b) after annealing, showing the change in stress from positive to negative curvature. The film thickness is $1 \mu \mathrm{m}$. 


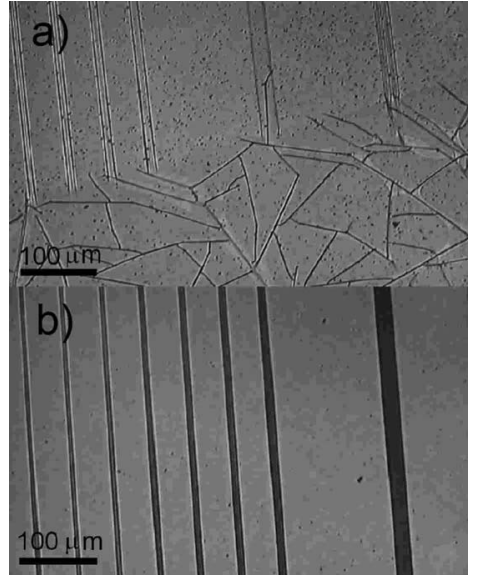

FIG. 3. Optical microscope images of Er:SRSO samples that have been etched into ridge structures prior to annealing: (a) shows a partly etched sample in which cracking is absent in the etched portion, but is clearly evident in the unetched section; (b) shows a fully etched sample-no cracking is evident.

We obtain stress values of $625 \mathrm{MPa}$ for the unannealed sample (compressive stress) and $-606 \mathrm{MPa}$ after annealing (tensile stress). These values agree well with reports in literature; ${ }^{12}$ such a large change in stress of $1.24 \mathrm{GPa}$ is clearly sufficient to cause film fracture.

Figure 3 shows microscope images of two test samples that have been prepared to prevent cracking: the lower half of the top picture is an area of the sample that was not etched, while the top half consisted of a series of ridges that were etched prior to annealing. Clearly visible are the ridge waveguides at the top of the picture, and severe cracks at the bottom. There is a little cracking at the interface of the two regions for the widest $(20 \mu \mathrm{m})$ of the waveguide structures, but the other waveguides are intact. Figure 3(b) shows a sample that has been completely etched, down to the substrate. In this case, all the waveguides are intact with no evidence of cracking. We find that even for very silicon-rich samples, etching the ridge structures down to the substrate completely eliminates stress-induced cracking.

However, significant uniaxial stress still exists along the axis of the etched ridges, as seen from Fig. 4, which shows a

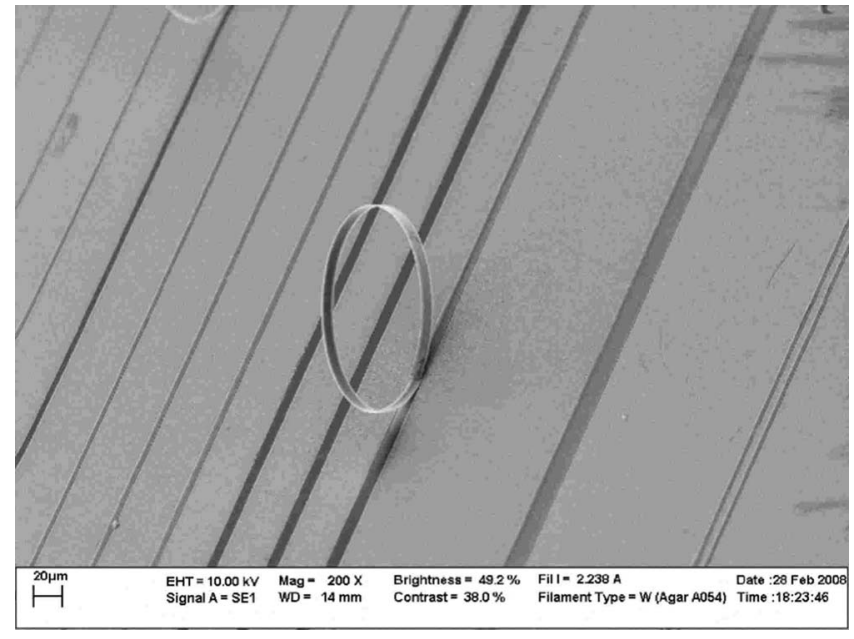

FIG. 4. An etched sample with poor film-substrate adhesion, showing curling of the etched ridge due to the presence of uniaxial stress in the ridge.
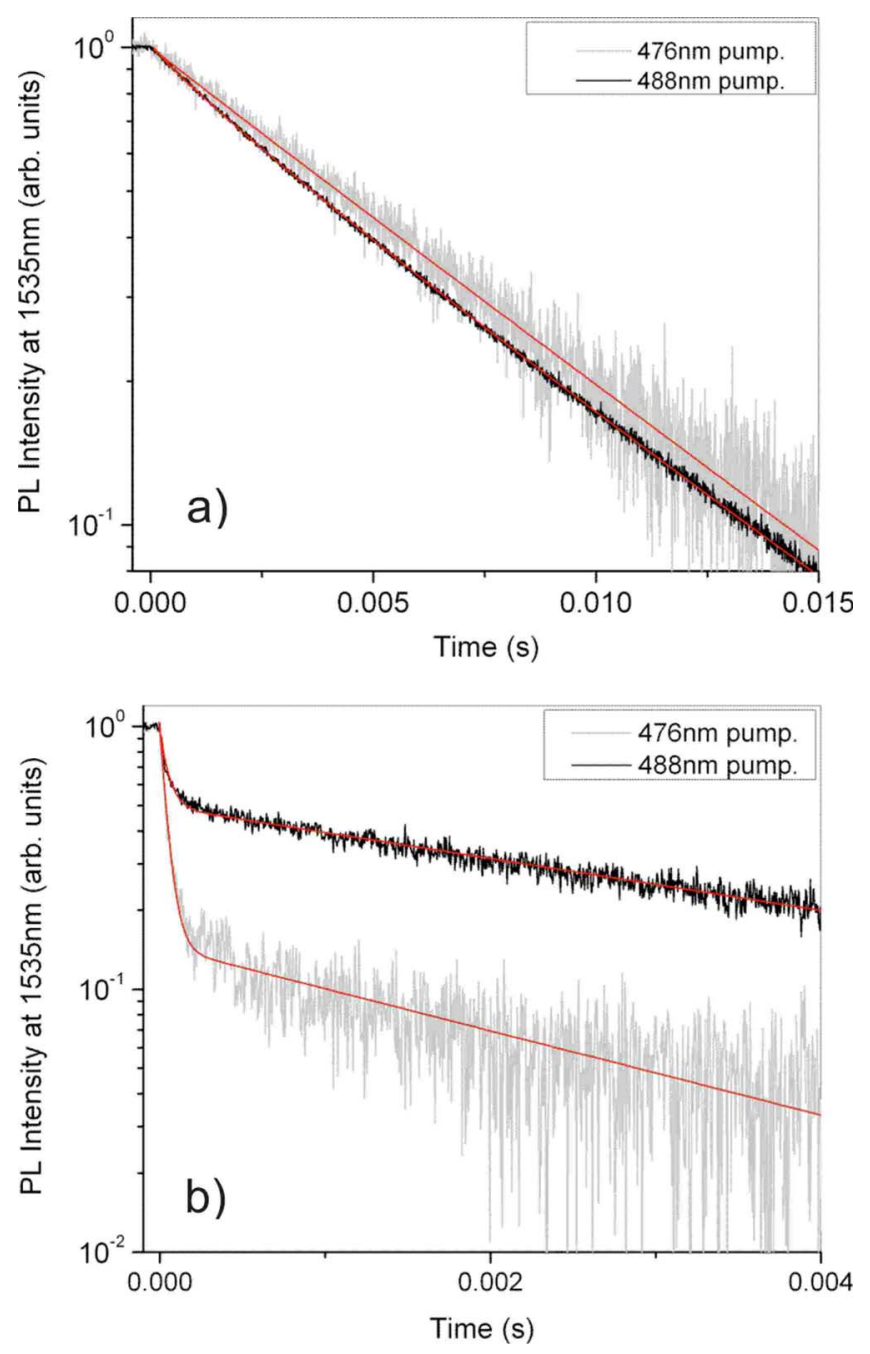

FIG. 5. (Color online) Er PL decays for (a) a portion of a sample that has cracked and peeled away from the substrate on annealing, and (b) a section of the same sample that remains adhered to the substrate. Note the different time axes. Decays are shown for pump wavelengths of $476 \mathrm{~nm}$ (nonresonant) and $488 \mathrm{~nm}$ (resonant). Solid lines are fits to the data.

sample where we have not subjected the substrate to Ar plasma surface treatment prior to film deposition. The film adhesion in this case is weak. This has enabled one of the ridges to partially detach from the substrate and form an upright and well-defined ring structure due to the internal stress still remaining in the ridge.

\section{THE EFFECT OF STRESS ON SILICON NANOCLUSTER FORMATION AND ERBIUM PHOTOLUMINESCENCE BEHAVIOR}

The presence of high stresses within the films has some surprising and quite significant consequences for Si-nc formation in the film, and the resulting sensitized erbium PL. Figure 5 shows the erbium PL decay (at $1535 \mathrm{~nm}$ ) recorded from (a) an area of an unetched film that has cracked and delaminated from the substrate due to biaxial stress, and (b) an area of the same film that has cracked but is still adhering to the substrate. The PL decay is markedly different in the two cases, despite having been grown on the same substrate at the same time, with the same PECVD deposition parameters. Where the film has delaminated, the erbium emission 


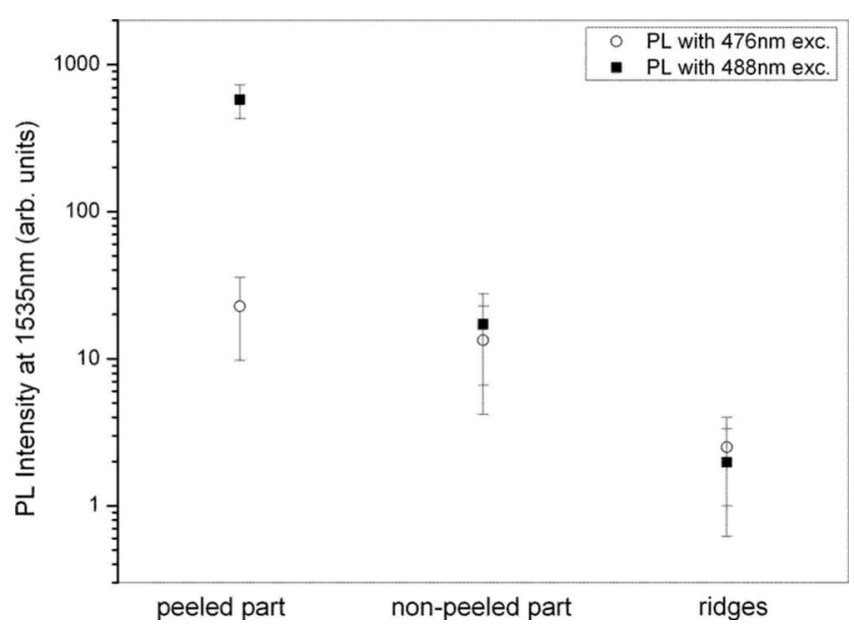

FIG. 6. Er PL peak intensities at $\lambda=1535 \mathrm{~nm}$ for sections of cracked, peeled, and ridged samples, pumped resonantly and nonresonantly.

exhibits a long lifetime of around $6 \mathrm{~ms}$, but where the film is still adhering to the substrate, the decay exhibits a short lifetime component of $\sim 40 \mu \mathrm{s}$, indicating that the erbium is strongly quenched in this latter case. We should note that the PL decays for the peeled section of film were best fitted using stretched exponential functions, although the values of beta were close to 1 ( 0.98 for $476 \mathrm{~nm}$ excitation, 0.91 for $488 \mathrm{~nm}$ excitation).

In fact, not only is the PL time decay behavior different between the delaminated and nondelaminated portions of the film, but PL intensities are also different when pumped at different wavelengths (476 and $488 \mathrm{~nm}$ ) wavelengths. From Fig. 6, we find that where the film has been etched or cracked but is still adhering to the substrate, the PL intensity is similar regardless of whether the pump wavelength is 476 or $488 \mathrm{~nm}$. In contrast, where the film is delaminated, the PL is much stronger (by more than an order of magnitude) under $488 \mathrm{~nm}$ pumping. The PL measurements were made over five to ten spots in each region and the variation found is reflected in the error bars. For the etched/ridged sample, the PL intensity is an order of magnitude lower than the other two samples simply because much of the film has been etched away.

To explain the PL behavior, we first note that erbium has no optical absorption band at the wavelength of $476 \mathrm{~nm}$. Therefore, with $476 \mathrm{~nm}$ pumping, it is the Si-ncs that are optically pumped; the $1535 \mathrm{~nm}$ PL comes from the sensitized erbium ions that are indirectly excited via the $\mathrm{Si}$ ncs. ${ }^{2}$ With 488 nm pumping, erbium can be directly optically excited since it has an absorption band at $488 \mathrm{~nm}$, as well as still being indirectly excited via the Si nc. From the data in Figs. 5 and 6, we can extract some very useful insights into the structure of the film, as described below.

First, from the ratio of the PL intensities under 488 and $476 \mathrm{~nm}$ pumping, we obtain an estimate of the fraction of $\mathrm{Er}$ ions that are indirectly excited via the Si-ncs. In the delaminated area of the film, the $488 \mathrm{~nm}$ pumped PL is measured to be $\sim 30$ times greater than that pumped at $476 \mathrm{~nm}$. Since the optical excitation cross section for $488 \mathrm{~nm}$ excitation is $\sim 1000$ times smaller than that of erbium excited indirectly via the $\mathrm{Si}$ ncs, ${ }^{21,22}$ the fraction of erbium ions that are indi-

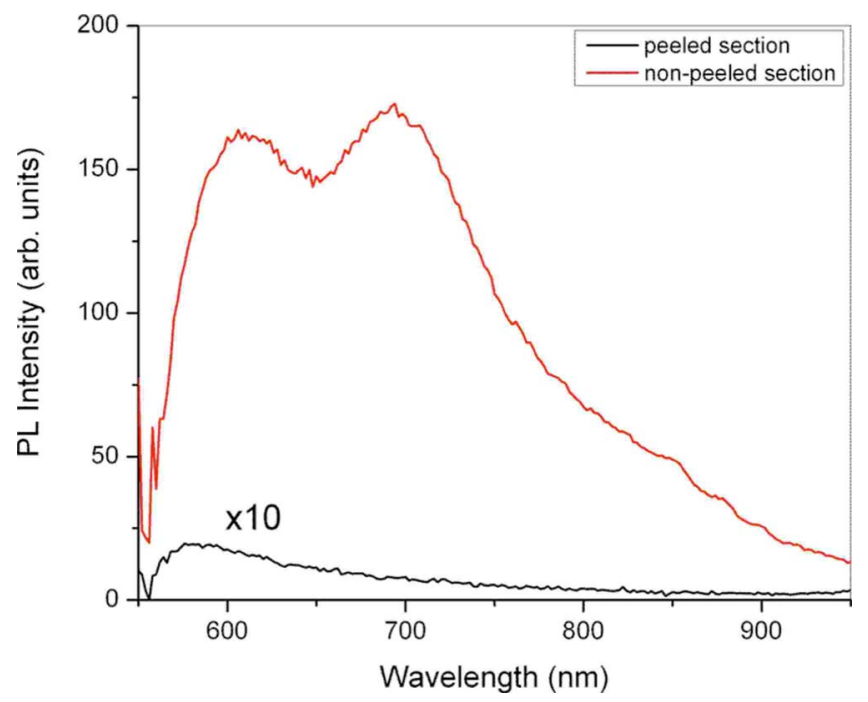

FIG. 7. (Color online) Visible and near-IR PL from peeled nonpeeled sections of a sample, showing Si nc PL from the unpeeled section. Pump wavelength is $476 \mathrm{~nm}$.

rectly pumped is therefore of the order $1 /(1000 \times 30)$ $=0.003 \%$ (assuming the same lifetime), a surprisingly small fraction.

For the areas of the sample where the film is cracked but still adhering to the substrate, the PL under $476 \mathrm{~nm}$ excitation is comparable in intensity to the delaminated sections. However, the decay lifetime is 150 times shorter, indicating that the erbium in this portion of the film is strongly quenched. To yield the same PL intensity, the fraction of indirectly excited $\mathrm{Er}$ must therefore be correspondingly greater: $0.003 \times 150 \% \%=0.45 \%$ of the erbium in the film. This fraction, while still low, is comparable to reports in previous studies of Er-doped silicon-rich silicon oxide (SRSO). ${ }^{23,24}$

Since the composition of the two sections of the film is the same, the two orders of magnitude difference that we find in the amount of indirectly excited Er in the same film suggests that the amount of Si nc precipitated into the film under annealing is not simply a function of the annealing temperature as often commonly assumed, but is likely due more to the stress encountered in the film while annealing. We note that recent results reported by La Magna et al. suggest a role for stress in phase separation of silicon in silicon-rich oxides. $^{25}$ By delaminating and enabling the film to curl away freely from the substrate, the film stress is relieved. The lack of sensitized erbium in the delaminated film indicates that very few Si ncs are actually precipitated under these conditions. To verify this, a broad wavelength PL scan was performed to directly capture the Si nc PL in the 400-1000 nm range under $476 \mathrm{~nm}$ pump. The results are shown in Fig. 7. Indeed, virtually no PL from the Si ncs could be detected in the case of the film flakes that had peeled away from the substrate. On the other hand, a broad and easily detectable spectrum suggestive of the presence of Si-ncs was observed for the cracked film that adhered to the substrate.

The Er PL time decay, Er PL intensity, and Si nc PL spectra presented here strongly suggest the presence of $\mathrm{Si}$ ncs in the stressed (adhered) film, but the virtual absence of 
nanoclusters in the relaxed (peeled) film, in spite of the films being deposited and annealed under identical conditions. The conventional picture of the formation of Si-ncs in siliconrich silica is that phase separation and consequent cluster nucleation and growth are thermally driven, and hence controlled by postdeposition annealing. Our results indicate that the picture may be more complex, and film stress plays a much larger role in the nucleation process than previously assumed. The mechanism by which the clustering of silicon is impeded by the presence of stress in the films is unclear at this stage, but we postulate that high internal stresses may promote the mobility of silicon atoms, allowing clusters to nucleate and grow. The evolution of hydrogen during annealing results in dangling bonds and microscopic voids that may promote the formation of clusters. However, such explanations remain speculative at present; further detailed studies of the microstructure of $\mathrm{SiO}_{x}$ films are required in order to develop a less phenomenological picture of the role of stress in nanocluster nucleation-such studies are outside the scope of the present work, but can be expected to yield important insights in to the microscopic behavior of this inhomogeneous material.

We note in addition that the phenomenon of nanoclustersensitized luminescence is a complex one, involving strongly distance-dependent interactions between sensitisers and rare earth ions. ${ }^{26}$ Because sensitizer-rare earth separations may be affected by local stresses, and the local crystal field surrounding the rare earth ions may also be affected by stress, the picture may be expected to be more complex than that presented here. However, Fig. 7 strongly suggests that the delaminated (stress relaxed) film does not contain any Si-ncs, and we thus discount changes in nanocluster-erbium separations or geometry as being responsible for the observed PL behavior. Moreover, rare earth ions are notoriously insensitive to the crystal field in which they are placed; shielding of the $4 f$ orbitals responsible for the optical activity of the rare earths by the $5 s^{2}$ and $5 p^{6}$ electrons means that transitions are only very weakly influenced by the local environment. ${ }^{27} \mathrm{We}$ postulate, therefore, that the change in the degree of sensitization as a result of stress arises predominantly from the change in the number of sensitisers, i.e., silicon nanoclusters.

\section{CONCLUSIONS}

In conclusion, thick $\mathrm{SiO}_{x}$ : Er layers grown by PECVD show that the films have large compressive stress before annealing, which changes to tensile stress after annealing, correlating with the release of hydrogen. As a consequence, thick films invariably crack from the high stresses suffered during annealing. We demonstrate that this cracking problem can be eliminated by patterning the material to allow relaxation of all film stress except for that along the axis of the ridges. However, significant stress remains along the ridge axis, as evidenced by ridge delamination in the case of reduced surface adhesion.
The presence of stress in these films has important consequences for the efficiency of sensitised erbium PL from silicon-rich material. Stressed films exhibit a higher degree of sensitization, along with PL from Si-ncs, but have rather short erbium PL lifetimes. We have found that stress promotes the formation of Si-ncs in silicon-rich material, which act as efficient sensitisers of erbium PL.

\section{ACKNOWLEDGMENTS}

The authors gratefully acknowledge financial support from the Engineering and Physical Sciences Research Council (EPSRC) in the UK, and the European Commission as part of the LANCER project (FP6-033574). One of the authors (C.J.O.) acknowledges support from an EIF Marie $\mathrm{Cu}-$ rie Fellowship under the EU 6th framework program. H.S. acknowledges support from the Daphne Jackson Memorial Trust.

${ }^{1}$ T. Shimizu-Iwayama, S. Nakao, and K. Saitoh, Appl. Phys. Lett. 65, 1814 (1994).

${ }^{2}$ A. J. Kenyon, P. F. Trwoga, M. Federighi, and C. W. Pitt, J. Phys.: Condens. Matter 6, L319 (1994).

${ }^{3}$ M. Fujii, M. Yoshida, Y. Kanzawa, S. Hayashi, and K. Yamamoto, Appl. Phys. Lett. 71, 1198 (1997).

${ }^{4}$ F. Iacona, D. Pacifici, A. Irrera, M. Miritello, G. Franzò, F. Priolo, D. Sanfilippo, G. Di Stefano, and P. G. Fallica, Appl. Phys. Lett. 81, 3242 (2002).

${ }^{5}$ J. Lee, J. H. Shin, and N. Park, J. Lightwave Technol. 23, 19 (2005).

${ }^{6}$ H.-S. Han, S.-Y. Seo, and J. H. Shin, Appl. Phys. Lett. 79, 4568 (2001).

${ }^{7}$ K. Watanabe, M. Fujii, and S. Hayashi, J. Appl. Phys. 90, 4761 (2001).

${ }^{8}$ F. Gourbilleau, C. Dufour, M. Levalois, J. Vicens, R. Rizk, C. Sada, F. Enrichi, and G. Battaglin, J. Appl. Phys. 94, 3869 (2003).

${ }^{9}$ L. A. Nesbit, Appl. Phys. Lett. 46, 38 (1985).

${ }^{10}$ S. Mani and T. M. Saif, Appl. Phys. Lett. 86, 201903 (2005).

${ }^{11}$ D. A. P. Bulla and N. I. Morimoto, Thin Solid Films 334, 60 (1998).

${ }^{12}$ R. G. Elliman, T. D. M. Weijers-Dall, M. G. Spooner, T.-H. Kim, and A. R. Wilkinson, Nucl. Instrum. Methods Phys. Res. B 249, 310 (2006).

${ }^{13}$ F. Ay and A. Aydinli, Opt. Mater. (Amsterdam, Neth.) 26, 33 (2004).

${ }^{14}$ K. Wörhoff, P. V. Lambeck, and A. Driessen, J. Lightwave Technol. 17, 1401 (1999).

${ }^{15}$ B. S. Sahu, O. P. Agnihotri, S. C. Jain, R. Mertens, and I. Kato, Semicond. Sci. Technol. 15, L11 (2000).

${ }^{16}$ G. Turban, Y. Catherine, and B. Grolleau, Thin Solid Films 77, 287 (1981).

${ }^{17}$ A. J. Kenyon, P. F. Trwoga, C. W. Pitt, and G. Rehm, J. Appl. Phys. 79, 9291 (1996).

${ }^{18}$ W. N. Sharpe, Jr., J. Pulskamp, D. S. Gianola, C. Eberl, R. G. Polcawich, and R. J. Thompson, Exp. Mech. 47, 649 (2007).

${ }^{19}$ E. Klokholm, IBM J. Res. Dev. 31, 585 (1987).

${ }^{20}$ G. G. Stoney, Proc. R. Soc. London, Ser. A 82, 172 (1909).

${ }^{21}$ G. Franzò, V. Vinciguerra, and F. Priolo, Appl. Phys. A: Mater. Sci. Process. 69, 3 (1999).

${ }^{22}$ A. J. Kenyon, C. E. Chryssou, C. W. Pitt, T. Shimizu-Iwayama, D. E. Hole, N. Sharma, and C. J. Humphreys, J. Appl. Phys. 91, 367 (2002).

${ }^{23}$ M. Wojdak, M. Klik, M. Forcales, O. B. Gusev, T. Gregorkiewicz, D. Pacifici, G. Franzò, F. Priolo, and F. Iacona, Phys. Rev. B 69, 233315 (2004).

${ }^{24}$ C. J. Oton, W. H. Loh, and A. J. Kenyon, Appl. Phys. Lett. 89, 031116 (2006).

${ }^{25}$ A. La Magna, G. Nicotra, C. Bongiorno, C. Spinella, M. G. Grimaldi, E. Rimni, L. Carista, and S. Coffa, Appl. Phys. Lett. 90, 183101 (2007).

${ }^{26}$ A. J. Kenyon, M. Wojdak, I. Ahmad, W. H. Loh, and C. J. Oton, Phys. Rev. B 77, 035318 (2008).

${ }^{27}$ A. J. Kenyon, Prog. Quantum Electron. 26, 225 (2002). 